Check for updates

Cite this: RSC Adv., 2019, 9, 25462

Received 16th March 2019 Accepted 19th June 2019

DOI: $10.1039 / c 9 r a 02026 h$

rsc.li/rsc-advances

\section{Bioceramic akermanite enhanced vascularization and osteogenic differentiation of human induced pluripotent stem cells in 3D scaffolds in vitro and vivo†}

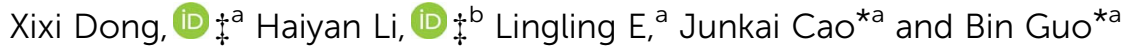

A growing number of studies suggest that the modulation of cell differentiation by biomaterials is critical for tissue engineering. In previous work, we demonstrated that human induced pluripotent stem cells (iPSCs) are remarkably promising seed cells for bone tissue engineering. In addition, we found that the ionic products of akermanite (Aker) are potential inducers of osteogenic differentiation of iPSCs. Furthermore, composite scaffolds containing polymer and bioceramics have more interesting properties compared to pure bioceramic scaffolds for bone tissue engineering. The characteristic of model biomaterials in bone tissue engineering is their ability to control the osteogenic differentiation of stem cells and simultaneously induce the angiogenesis of endothelia cells. Thus, this study aimed at investigating the effects of poly(lactic-co-glycolic acid)/Aker (PLGA-Aker) composite scaffolds on angiogenic and osteogenic differentiation of human iPSCs in order to optimize the scaffold compositions. The results from Alizarin Red S staining, qRT-PCR analysis of osteogenic genes (BMP2, RUNX2, ALP, COL1 and OCN) and angiogenic genes (VEGF and CD31) demonstrated that PLGA/Aker composite scaffolds containing $10 \%$ Aker exhibited the highest stimulatory effects on the osteogenic and angiogenic differentiation of human iPSCs among all scaffolds. After the scaffolds were implanted in nu/nu mice subcutaneous pockets and calvarial defects, H\&E staining, BSP immunostaining, qRT-PCR analysis and micro-CT analysis (BMD, BV/TV) indicated that PLGA $+10 \%$ Aker scaffolds enhanced the vascularization and osteogenic differentiation of human iPSCs and stimulated the repair of bone defects. Taken together, our work indicated that combining scaffolds containing silicate bioceramic Aker and human iPSCs is a promising approach for the enhancement of bone regeneration.

\section{Introduction}

Induced pluripotent stem cells (iPSCs) have been widely used as seed cells in bone tissue engineering because they present high similarity with embryonic stem cells (ESCs), can be easily generated from somatic cells, and their use can avoid immunological rejection and is exempt from ethical restrictions. ${ }^{1-3}$ Bilousova et al. reported that osteoblasts derived from iPSCs can form calcified structures in three-dimensional (3D) scaffolds both in vitro and in vivo. ${ }^{4}$ Moreover, numerous studies have demonstrated that iPSCs or modified iPSCs seeded on 3D scaffolds improve bone regeneration and the repair of bone

\footnotetext{
${ }^{a}$ Stomatology Department, General Hospital of Chinese PLA, 28 Fu Xing Road, Beijing 100853,China.E-mail: caojk301@163.com; guobin0408@126.com

${ }^{b}$ Med-X Research Institute, School of Biomedical Engineering, Shanghai Jiao Tong University, 1954 Huashan Road, Shanghai 200030, China

$\dagger$ Electronic supplementary information (ESI) available. See DOI: 10.1039/c9ra02026h

\$ These authors contributed equally.
}

defects in vivo..$^{5-7}$ Thus, studies on the incorporation of iPSCs in tissue engineering are highly encouraged.

In a previous work, we found that silicon ( $\mathrm{Si}$ ) ions released from silicate-based bioceramic Akermanite (Aker) enhance the osteogenic differentiation of human iPSCs. ${ }^{8}$ This finding signposted the application potential of Aker scaffolds as suitable carriers of iPSCs in bone tissue engineering. However, given that bone tissue is an organic/inorganic composite, threedimensional (3D) scaffolds with the organic/inorganic composites display obvious advantages compared to pure organic scaffolds. ${ }^{9-14}$ Among these scaffolds, polyester/silicate bioceramic composite scaffolds have attracted particular attention in the field of bone regeneration ${ }^{15-17}$ owing to the excellent bioactivity of silicate ceramics and the good biocompatibility and degradability of polyesters. ${ }^{18-20}$ On the one hand, the inorganic part of composites, including bioceramic calcium silicate (CS), bioglass (BG) and Aker, could induce hydroxyapatite deposition on scaffolds, which is a critical factor for enhancing bone regeneration. On the other hand, the bioceramics in composite scaffolds could release $\mathrm{Ca}$ and $\mathrm{Si}$ ions, 
which could not only stimulate osteogenic differentiation of different stem cells ${ }^{15-17,21-23}$ but also balance the acidic microenvironment caused by the degradation of organic materials. ${ }^{10-14}$ Therefore, it is advantageous to incorporate Aker in composite scaffolds during bone tissue engineering. However, studies focused on the incorporation of Aker in composite scaffolds are limited and in-depth studies are needed to elucidate and validate Aker-based composite scaffolds.

Angiogenesis is a key challenge in bone regeneration. Previous studies demonstrated that silicate-based bioceramics, including CS and BG could stimulate angiogenesis. ${ }^{15,16,24-28}$ In a previous study, we fabricated an organic/ inorganic composite scaffold poly(lactic-co-glycolic acid) PLGA/calcium silicate (CS), and found that the composite scaffolds could significantly enhance the osteogenic and angiogenic differentiation of human bone marrow stromal cells (HBMSCs) and human umbilical vein endothelial cells (HUVECs). ${ }^{15}$ Aker ionic products and its bioceramic scaffolds have also been reported as enhancers of the angiogenic differentiation of human aortic endothelial cells (HAECs) in bone regeneration. ${ }^{\mathbf{2 9 , 3 0}}$ Furthermore, the degradable polylactic acid composite scaffold (PDLLA)/Aker has been also reported to enhance the angiogenesis of HUVECs. ${ }^{17}$ The above evidences show that the Aker bioceramics are able to induce angiogenesis. However, the effect of Aker on the angiogenic differentiation of iPSCs has not been reported so far. This is a new interesting frame to explore in bone tissue engineering.

The 3D porous structure of the composite scaffolds offers cells 3D environment, and prevent cells to flow away from targeted site while allowing nutrients and oxygen to be diffused in, and wastes to be discharged out. ${ }^{31}$ Moreover, the incorporation of inorganic particles into polymers could produce suitable hydrophilicity scaffolds with satisfactory properties for cell adhesion and proliferation. ${ }^{\mathbf{1 0 - 1 4}} \mathrm{Up}$ to now, there is no wellcharacterized engineered 3D porous iPSCs-carrying composite scaffold that is able to simultaneously induce both the angiogenic and osteogenic differentiation of iPSCs. In addition, the function and behavior of iPSCs in bone repair is not well elucidated.

Therefore, in this study, we aimed to fabricate PLGA/Aker composite scaffolds and investigate whether the Aker in composite 3D scaffolds can stimulate the angiogenic and osteogenic differentiation of human iPSCs in vitro and in vivo. The ultimate goal is to obtain a suitable engineered iPSCs-carrying scaffold for bone repair.

\section{Experimental}

\subsection{Cell culture}

The human iPSCs were purchased from Beijing Cellapy Biotechnology (China). Human iPSCs were cultured on Matrigel-coated dishes (BD Biosciences, Canada) with PSCeasy medium (Beijing Cellapy Biotechnology, China). Diffeasy medium (Beijing Cellapy Biotechnology, China) was used in differentiation studies.

\subsection{Preparation of PLGA/Aker composite scaffolds and in vitro cell seeding}

The akermanite powder was prepared as described previously. ${ }^{32}$ Poly(lactic-co-glycolic acid) (PLGA, 75 : 25 molar ratio, $M_{\mathrm{w}} 1 / 450$ $\mathrm{kDa}$ ) was purchased from Jinan Daigang Co. (Jinan, China). Previous studies showed PLGA does not disturb the osteogenesis of iPSCs. ${ }^{33}$ Aker particles with size ranging from 100 to $150 \mu \mathrm{m}$ were used to prepare the PLGA/Aker composite scaffolds with two different weight ratios of CS: PLGA $+10 \%$ Aker and PLGA $+20 \%$ Aker according to our previous studies. ${ }^{11,14,15}$ The pure PLGA and composite scaffolds were prepared by solvent casting particle leaching method reported in literature. ${ }^{\mathbf{1 2}}$

Before cells were seeded, scaffolds were cut into disk-shape, $4 \mathrm{~mm}$ in diameter and $2 \mathrm{~mm}$ in thickness. The scaffolds were immersed into $75 \%$ ethanol for $2 \mathrm{~h}$, followed by rinsing with PBS five times. Then, scaffolds were placed in 24 -well plates. $2 \times$ 107 human iPSCs seeded in presence of the three different groups of scaffolds: PLGA, PLGA $+10 \%$ Aker and PLGA $+20 \%$ Aker. Cell attachment and cell growth were assessed at day 5 after cell seeding. The constructs were fixed in $4 \%$ paraformaldehyde (PFA). After thoroughly washing with PBS, the cells adhered to the scaffold section and subsequently dehydrated in gradient concentrations of ethanol (50-100\%) for 10 min each, and allowed to dry on a clean bench at room temperature. ${ }^{34}$ The porous structure of scaffolds and the state of cells were examined with a scanning electron microscope (SEM, FEI, QUANTA 250, Netherlands) using an accelerating voltage of $10 \mathrm{kV}$.

\subsection{In vitro evaluation of angiogenic and osteogenic differentiation on scaffolds}

2.3.1 Alizarin Red S staining. Alizarin Red S staining was performed to analyze the newly formed nodules on the scaffolds. Briefly, after culture in differentiation medium (Beijing Cellapy Biotechnology, China) for 21 days, the three scaffolds were taken out from each group. Next, cells on the scaffolds were washed twice with PBS and with 4\% PFA for $20 \mathrm{~min}$ at room temperature, followed by several washes with distilled $\mathrm{H}_{2} \mathrm{O}$. Thereafter, cells were stained with $2 \%(\mathrm{w} / \mathrm{v})$ Alizarin Red (Sigma) at room temperature for $5 \mathrm{~min}$ and subsequently washed several times with distilled $\mathrm{H}_{2} \mathrm{O}$.

2.3.2 Quantitative real-time polymerase chain reaction (qRT-PCR). After seeding on scaffolds and culturing of cells in differentiation medium for 2 weeks, qRT-PCR was performed to evaluate the expression of angiogenic and osteogenic genes of the human iPSCs on the 3D scaffolds as described previously. ${ }^{8}$ We used the E.Z.N.A Total RNA Kit I (Omega, Bio-tek) to isolate total RNA from cells cultured in different groups according to the manufacturer's instructions. The concentration was measured using a nanodrop 1000 reader (Thermo Scientific). The ReverTra Ace-a kit (Toyobo Co., Ltd, Japan) was used to synthesize the cDNA following the manufacturer's recommendations. Then we performed qRT-PCR reactions using SYBRGreen Master Mix (Toyobo Co., Ltd). Primer sequences (Table S1) (ESI $\dagger$ ) for RUNX2, BMP2, ALP, COL1, OCN, VEGF, CD31 amplification were synthesized by Sangon Biotech Co., Ltd 
(Shanghai). Data were normalized to GAPDH mRNA expression of each condition and were quantified relative to the corresponding gene expressions from control samples, which were standardized to 1 .

\subsection{In vivo implantation of cell/scaffold}

2.4.1 In vivo subcutaneous transplantation. All surgical procedures were performed in accordance with the NIH guidelines for the care and use of laboratory animals (NIH publication no. 86-23 rev. 1985) and was approved by IRB of our Chinese PLA General Hospital. After culture in differentiation medium for 2 weeks in vitro, cell-containing scaffolds were implanted into subcutaneous pockets in the dorsal region of 6-8 week old nu/nu mice purchased from the Chinese PLA General Hospital Animal Center. 4 pockets were made on each mouse and respectively inserted with the 4 different implants (PLGA, PLGA $+10 \%$ Aker, PLGA + C, PLGA $+10 \%$ Aker $+\mathrm{C})(\mathrm{C}$ represent the Human iPSCs $)$. The pockets on dorsal region of each mouse were made according to previously described methods. ${ }^{35}$ Briefly, mice were firstly anesthetized until numbness. Next, a $2 \times 2 \mathrm{~cm}$ area on the back was shaved and the area wiped with gauze soaked with $75 \%$ ethanol. After that, a small (about $1.5 \mathrm{~cm}$ ) longitudinal incision was made into the skin at the center of the shaved area and the tips of dissecting scissors were used to dissect subcutaneous facial tissue and create pockets under the skin (between the skin and muscle layers). Finally, different cell-containing scaffolds or scaffolds without cells were implanted into the pockets and the incisions were sutured. For preventing the mice from chewing on the sutures, a tissue adhesive was used to cover the closed wound. Animals were then put under a heat lamp for $1 \mathrm{~h}$ to allow them recover postoperatively before being returned to their cages. At 4 and 8 weeks, animals were killed and implants were analyzed for blood vessel formation and osteogenic differentiation.

2.4.2 Analysis of in vivo blood vessel formation and osteogenesis. Implants were taken out at various time points and prepared for histological and immunohistochemical examination. The specimens were first fixed in $10 \%$ buffered formalin in PBS for 4-6 h, embedded in paraffin and then cut into $5 \mathrm{~mm}$ longitudinal sections. The sections were collected on glass slides for histological analysis. Tissue sections were stained with Gill's 3 hematoxylin (Sigma-Aldrich) and aqueous eosin Y solution (Sigma-Aldrich) (H\&E) to visualize the overall tissue morphology. Detection of human bone sialoprotein (BSP) was performed using an anti-human BSP antibody (Invitrogen) as described previously. ${ }^{35}$ The stained slides were finally counterstained with hematoxylin and dehydrated, mounted, and covered with coverslips. The percentage of the brown area to the total area of the BSP stained images were recorded as described previously. ${ }^{15}$ Meanwhile, the expression of gene CD31 and BSP in implants was analyzed by qRT-PCR as described above.

2.4.3 Restoration of critical-size bone defect. After the scaffolds and cells were incubated in the differentiation medium for 2 weeks, the cells/scaffolds were implanted into the bone defects, and the critical-size bone defects were made as mentioned in a previous study. ${ }^{5}$ All surgical procedures were performed in accordance with the NIH guidelines for the care and use of laboratory animals (NIH publication no. 86-23 rev. 1985) and was approved by IRB of our Chinese PLA General Hospital. Briefly, the nu/nu mice were anesthetized, and a $4 \mathrm{~mm}$ diameter calvarial critical-sized defect was created on each side of the calvarial bone using a dental bur attached to a slow-speed hand piece with minimal invasion of the dura mater. The critical-sized defects in mice were randomly divided into 5 groups to receive the following implants: (1) control (without cells and scaffolds) $(n=7)$; (2) PLGA $(n=7)$; (3) PLGA + Aker $(n=7)$; (4) PLGA + C $(n=7) ;(5)$ PLGA + Aker + C $(n=7)$. The mice were sacrificed 8 weeks post-surgery. The qRT-PCR experiment was used to detect the expression of osteogenic genes including RUNX2, BMP2, COL1 and OCN while immunostaining was performed to detect the expression of BSP. The qRT-PCR and immunostaining experiments were performed as described above.

2.4.4 Micro-CT measurement of critical-size bone defect. After cells/scaffolds implantation for 8 weeks in bone defect, micro-CT measurement was performed to evaluate the healing of calvarial defects. The specimens of 8 weeks after the surgical procedure were monitored using a Quantum FX microcomputed tomography imaging system (micro-CT, Caliper, USA). Field of view (FOV) scanning at $36 \mathrm{~mm}$ and a $4.5 \mu \mathrm{m}$ voxel size resolution were selected. The calculated bone mineral density (BMD) and $\mathrm{BV} / \mathrm{TV}$ in this area were presented as the percentage of defect regeneration. ${ }^{36,37}$ The parameters of bone volume fraction (bone volume/total volume, BV/TV) and bone mineral densities (BMD) were used for comparison in this study.

\subsection{Statistical analysis}

All analyses were performed in triplicate samples for $n=3$ at least. The qRT-PCR experiment was also performed on triplicate samples $(n=3)$ with triplicate readings. All the data were expressed as mean \pm standard deviation (SD). Differences between groups were analyzed by ANOVA (SPSS, v.17.5, USA). Significant difference was considered when $p<0.05$.

\section{Results}

\subsection{Growth of human iPSCs on 3D scaffolds}

The 3D scaffolds and cellular attachment and interaction within scaffolds were analyzed using the electron microscope after cells were grown in the scaffolds for 5 days. The results were depicted in Fig. 1 and showed that all cell-containing scaffolds were obviously porous. The pore size ranged from 20 to $300 \mathrm{~mm}$ (Fig. 1A). As shown in Fig. 1B, the cells grew tightly to each other and abundant extracellular matrix was deposited on the scaffolds. This indicated that the cell-containing scaffold was suitable for the subsequent in vivo studies given that it facilitated the initial attachment of cells onto the surface, their spreading and subsequent growth.

\subsection{Angiogenic and osteogenic differentiation in 3D scaffolds}

After cells were seeded in scaffolds for 21 days, alizarin red S was used to evaluate the degree of mineralization of the cell- 


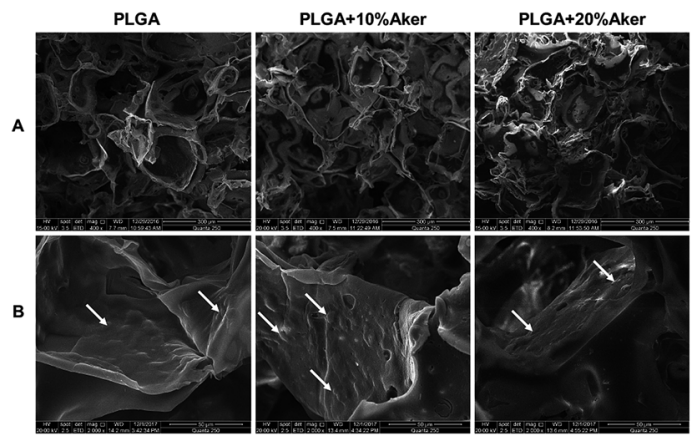

Fig. 1 Scanning electron microscopic evaluation of the scaffold microstructure and the state of cells. (A) Scaffold without cells; (B) 5 days after the human iPSCs were seeded onto the scaffold, cells (arrows) grew tightly to each other and some extracellular matrix deposited on the scaffold.

containing scaffolds. As shown in Fig. 2A, the results of alizarin red $S$ staining revealed matrix mineralization of the scaffolds. The PLGA + 10\% Aker and PLGA + 20\% Aker exhibited strongly positive staining reflecting the highly active matrix mineralization compared to the PLGA. Moreover, the extent of mineralization in the PLGA $+10 \%$ Aker group was stronger than that of the PLGA $+20 \%$ Aker group. This indicated the induction of osteogenesis in the PLGA $+10 \%$ Aker group. In order to evaluate the expression of osteogenic markers in cells attached to the scaffolds, qRT-PCR was performed after induction of the differentiation for 2 weeks. The expression of osteogenic markers such as BMP2, RUNX2, ALP, COL1 and OCN were enhanced in the PLGA $+10 \%$ Aker group compared to the PLAG and PLGA $+20 \%$ Aker groups (Fig. 2B).

In order to evaluate the angiogenic capability of the scaffolds, the expression of angiogenic genes including VEGF and CD31 was also determined. Similarly to the osteogenic markers, the expression of angiogenic genes were enhanced in PLGA + $10 \%$ Aker group compared to the PLAG and PLGA $+20 \%$ Aker (Fig. 2C).

These results suggest that $10 \%$ Aker could stimulate both angiogenic and osteogenic differentiation of human iPSCs in 3D scaffolds. Based on these in vitro results, we chose the PLGA and PLGA $+10 \%$ Aker scaffolds for further in vivo animal experiments.

\subsection{Angiogenic and osteogenic differentiation in subcutaneous pockets}

As shown in Fig. 3A, the animals subcutaneously bearing the PLGA + C (Fig. 3A-a) and PLGA + Aker + C (Fig. 3A-b) implants after 8 weeks implantation. The vascularization of the implants PLGA + Aker + C at 4 weeks (Fig. 3A-c) and 8 weeks (Fig. 3A-d) was obvious. The H\&E staining (Fig. 3B) of the implants 4 and 8 weeks after implantation was performed to evaluate the vascularization degree. The result showed a greater number of new formed blood vessels in the PLGA + Aker + C composite scaffolds compared to the other groups at both 4 and 8 weeks. Red blood cells (red circles) were also clearly observed. Furthermore, the expression of angiogenic gene CD31 was detected by qRT-
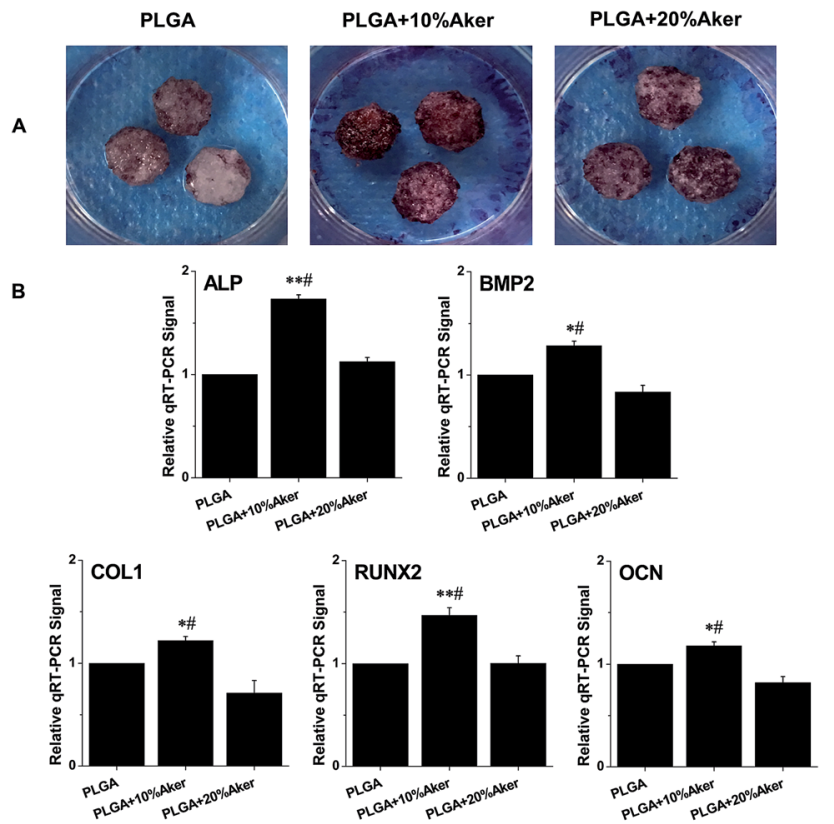

c

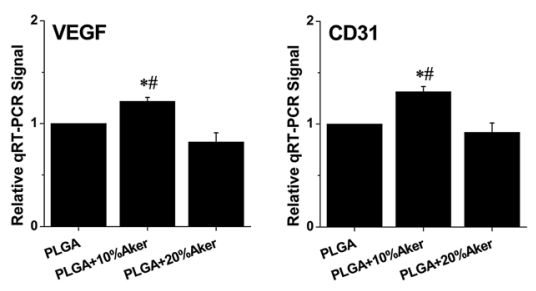

Fig. 2 The vascularization and osteogenic differentiation of human iPSCs in the 3D scaffolds. (A) Alizarin Red staining of human iPSCs indicating the formation of calcium nodules after culturing in different scaffolds for 21 days. (B) Quantitative real-time PCR was performed for expressions of osteogenic genes, such as ALP, BMP2, COL1, OCN and RUNX2 of human iPSCs in 3D scaffolds. (C) Quantitative real-time PCR was performed for expressions of angiogenic genes, including VEGF and CD31 of human iPSCs in 3D scaffolds. $* p<0.05, * * p<0.01$, compared with PLGA. ${ }^{*} p<0.05$ compared with PLGA $+20 \%$ Aker.

PCR and the results showed similar trends both at 4 and 8 weeks. Specifically, the expression of CD31 in the PLGA + Aker + $\mathrm{C}$ was the highest among all the groups (Fig. 3C). These results suggest that Aker in 3D scaffolds could stimulate the vascularization of human iPSCs in vivo.

Meanwhile, BSP staining was performed at two time points, 4 and 8 weeks (Fig. 4A). The result showed that the number of brown-stained granules were higher in the PLGA + Aker + C group than the other groups. Less brown structures were observed in the PLGA group. Moreover, QRT-PCRthe qRT-PCR results showed that the expression of BSP was higher in the PLGA + Aker + C group than the other groups at 4 and 8 weeks (Fig. 4B). These results suggested that Aker in 3D scaffolds could stimulate the osteogenic and angiogenic differentiation of human iPSCs in vivo.

\subsection{Bone healing of calvarial defects}

Defects of $4 \mathrm{~mm}$ in diameter were made in both sides of the cranial bone (Fig. 5A-a) and filled with cell-containing scaffolds (Fig. 5A-b). After 8 weeks the mice were sacrificed. Fig. 5A-c 
A
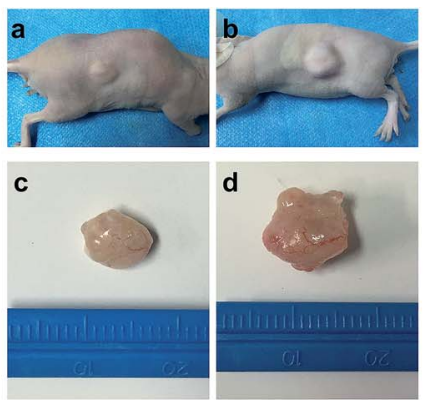

B

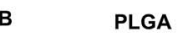

PLGA+Aker

PLGA+C

PLGA+Aker+C

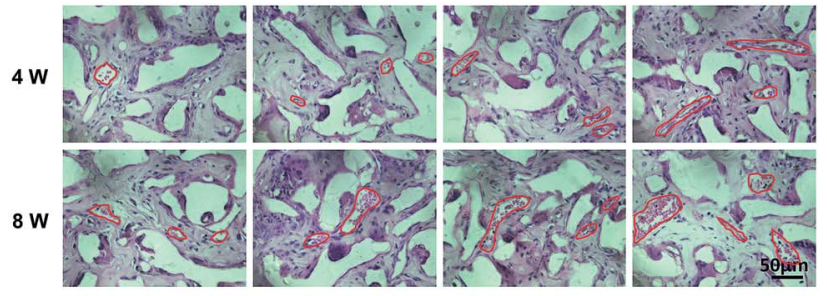

C

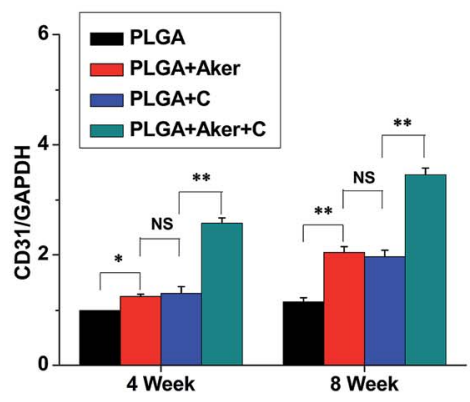

Fig. 3 The vascularization of human iPSCs in scaffolds in vivo. (A) (A-a) PLGA + C; (A-b) PLGA + 10\% Aker + C; PLGA + 10\% Aker + C group at 4 $(A-C)$ and $8(A-d)$ weeks after human iPSCs in scaffolds were implanted into subcutaneous pockets, implants were taken out for analysis. (B) H\&E staining, functional blood vessels were defined by structures that had a clearly defined lumen containing red blood cells (red circles), blood vessel of different implants at 4 and 8 weeks, bar $=50 \mu \mathrm{m}$. (C) Quantitative real-time PCR was performed for evaluating the expressions of angiogenic genes CD31 of the new formation tissue in vivo at 4 and 8 weeks. ${ }^{*} p<0.05, * * p 0.01$. NS: no significance.

shows the result of the control group in which the defects were not filled with scaffolds. The results of other groups were shown in Fig. 5A-d-g. As shown in Fig. 5A-c-g, it is observed that all the bone defects were healed. BSP staining (Fig. 5B) indicated that the PLGA + Aker + C group displayed the brownest stained area compared to the other groups. The least stained brown granules were found in the PLGA group compared with the other groups. The percentage of brown area (Fig. 5C) was the highest in the PLGA + Aker + C group, which indicated the highest BSP expression level. The expression level (percentage of brown area) of BSP was lower in PLGA group than other groups. In the meanwhile, there was no significant difference between PLGA + $\mathrm{C}$ and PLGA + Aker groups. Furthermore, the expression levels of osteogenic genes BMP2, RUNX2, COL1 and OCN detected by qRT-PCR were higher in PLGA + Aker + C group than the other groups (Fig. 5D), which indicated the osteogenesis driven by this implant.

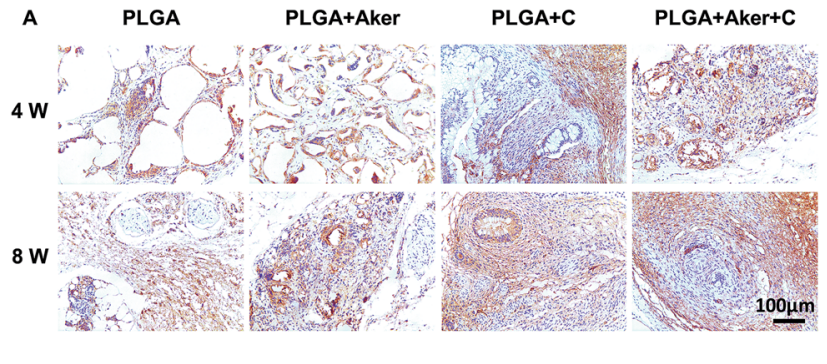

B

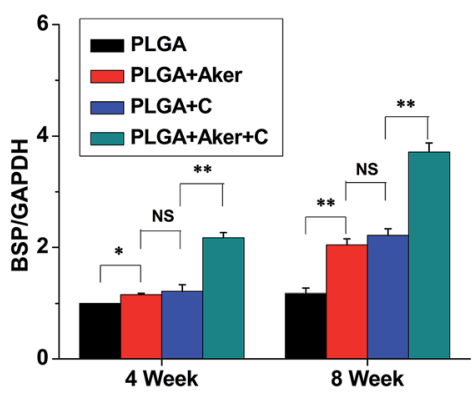

Fig. 4 The osteogenic differentiation of human iPSCs in scaffolds in vivo. (A) Implants were taken out from subcutaneous pockets at 4 and 8 weeks, the BSP immunostaining (brown structures) was performed to indicate the osteogenic differentiation of human iPSCs in vivo, bar = $100 \mu \mathrm{m}$. (B) Quantitative real-time PCR was performed for evaluating the expression of the osteogenic gene BSP in the newly formed bone tissue in vivo at 4 and 8 weeks. ${ }^{*} p<0.05, * *<0.01$. NS: no significance.

Micro-CT was used to detect the formation of new bone around the defects border after 8 weeks. The results are depicted in Fig. 6A and indicated that the majority of the cranial defects were filled with substantial newly formed bone tissue in the defect site treated with the PLGA + Aker + C implants. But no obvious evidence of new bone formation was observed in control and PLGA group, and minor evidence of new bone formation was visible in the periphery of the defect edges in PLGA + Aker and PLGA + C groups. To quantify the new bone formation within the calvarial defects, local bone mineral density (BMD) and the ratio of bone volume to total volume (BV/ TV) were measured. As shown in Fig. 6B, BMD for PLGA + Aker + C group was higher than the other groups, and BV/TV (Fig. 6C), as an indicator of the relative amount of newly formed bone, was significantly greater for the PLGA + Aker + C group when compared to all other groups. These results suggested that Aker could stimulate the osteogenic differentiation of human iPSCs and the formation of new bone within defects sites in vivo.

\section{Discussion}

It has been widely accepted that tissue engineering includes three factors: seed cells, scaffolds and growth factor. ${ }^{38-41}$ The potential of iPSCs seeded on 3D scaffolds for bone regeneration in vivo has been confirmed in several animal studies. ${ }^{4-7}$ However, interactions between the cell and its environment determine cell fate due to mechanical and biochemically secreted factors. ${ }^{42}$ Biomaterials, as carriers of seeded cells in 
A

B
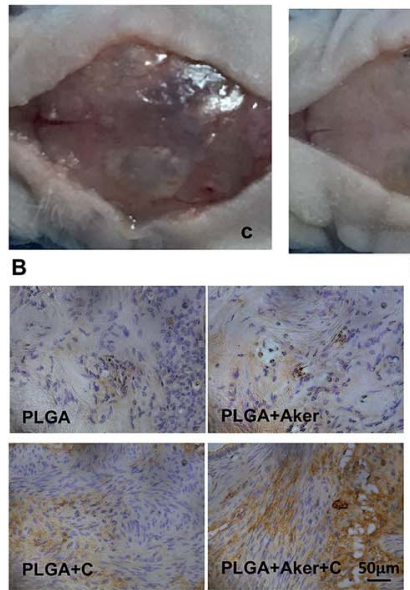
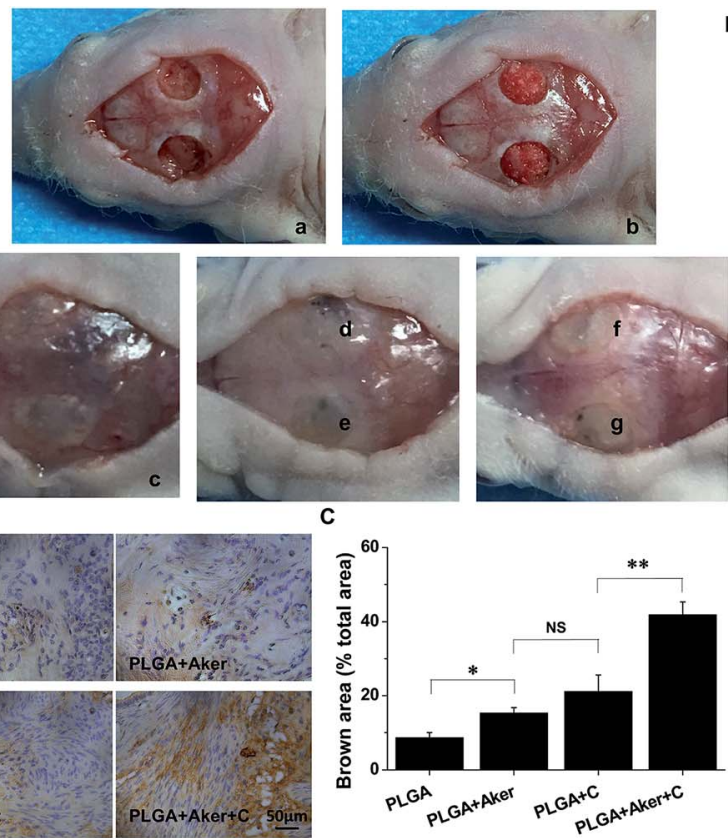

D
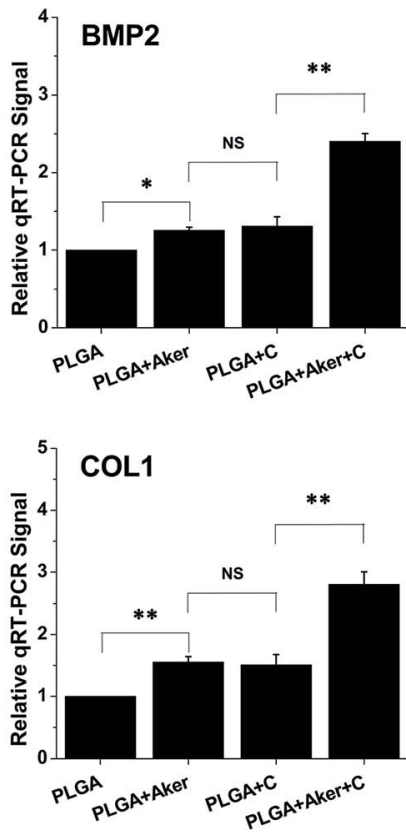
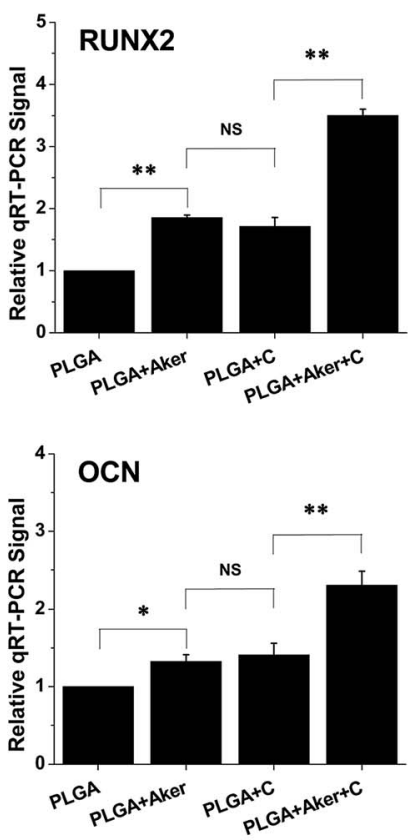

Fig. 5 Scaffolds combine Aker and human iPSCs promote the healing of skull defects. (A) Human iPSCs in scaffold complex in vivo for repairing skull defects. (A-a) A non-healing full thickness defect of $4 \mathrm{~mm}$ diameter in the either side of the cranial bone was made; (A-b) the skull defect was filled with an implant; (A-C-g) the skull defect was filled with nothing, PLGA, PLGA + C, PLGA + Aker and PLGA + Aker + C respectively after implantation for 8 weeks; (B) BSP secreted by cells was detected by immunostaining (brown structures) at 8 weeks, greater BSP expression was observed in the PLGA + Aker + C group than in the other groups. Bar $=50 \mu \mathrm{m}$; (C) the percentage of the brown area surface to the total surface of images taken from groups. The group PLGA + Aker + C secreted more BSP than the other groups. (D) Quantitative real-time PCR was performed for expressions of osteogenic marker genes BMP2, RUNX2, COL1 and OCN in new formation tissue around the defects. * $p<0.05, * * p<0.01$. NS: no significance.

bone tissue engineering, also play a vital role in regulating the growth and differentiation of stem cells. Recent studies have revealed that silicate bioceramics stimulate the osteogenic differentiation of stem cells, including bone marrow mesenchymal stem cells ${ }^{15-17,21-23,43,44}$ and urine-derived stem cells. ${ }^{45}$ Furthermore, studies also showed that silicate bioceramic are able to enhance angiogenesis of human umbilical vein endothelial cells (HUVECs), ${ }^{15,44}$ which is as important as osteogenesis in bone tissue engineering.

Recent studies have reported that bioceramic Aker can stimulate the osteogenesis of BMSCs ${ }^{29,30,46,47}$ and adipose-derived stem cells (ASCs) ${ }^{48,49}$ and the angiogenesis of hAECs. ${ }^{29,30}$ Moreover, our previous study indicated that $\mathrm{Si}$ ions released from Aker induces the osteogenic differentiation of human iPSCs in vitro. ${ }^{8}$ In the present study, we demonstrated that the stimulatory effects of PLGA $+10 \%$ Aker 3D scaffolds on angiogenic and osteogenic differentiation of human iPSCs was much higher comparatively to those of PLGA alone and PLGA $+20 \%$ Aker scaffolds. A previous study demonstrated that pure PLGA, as biodegradable polymers, lacks of bioactivity. ${ }^{50}$ This suggests that the PLGA cannot stimulate cell differentiation. Furthermore, the release of acidic degradation by-products from PLGA can lead to inflammatory responses. ${ }^{51-53}$ Moreover, the $\mathrm{Ca}$ and $\mathrm{Si}$ ions released from composites can neutralize the acidic degradation by-products of the PLGA. ${ }^{10-14}$ Thus, we speculated that the concentration of $20 \%$ Aker may be too much in 3D scaffold, which might cause high $\mathrm{pH}$ and subsequently reduce its effectiveness on the differentiation of iPSCs.
Furthermore, we also revealed that the composite scaffolds PLGA + Aker + C induced the formation of blood vessels and osteogenesis in subcutaneous pockets, and significantly repaired the defects comparatively to other groups. On the one hand, Aker is a Si-, Ca- and Mg-containing bioactive ceramic. Recent studies showed that $\mathrm{Si}-$, $\mathrm{Ca}-$ and Mg-containing ionic products from bioceramics could stimulate the osteogenic and angiogenic differentiation of BMSCs and HAVECs. ${ }^{29}$ Other studies have shown that $\mathrm{Si}$ - and Ca-containing ionic products from calcium silicate $\left(\mathrm{CaSiO}_{3}\right)$ bioceramics could also induce the osteogenesis of BMSCs and angiogenesis of HUVECs. ${ }^{15,16}$ Moreover, studies also reported that $\mathrm{Mg}$ can enhance bone growth and boost local blood perfusion compared to other metallic or polymeric materials. ${ }^{54-57}$ Therefore, it is reasonable to speculate that the $\mathrm{Si}$ and $\mathrm{Mg}$ ions released from composite scaffold PLGA + Aker in 3D scaffold are among the main factors improving their osteogenic and angiogenic differentiation ability. On the other hand, the osteogenesis and angiogenesis potential of iPSCs in 3D scaffolds and in vivo have been reported. ${ }^{4-7,58-67}$ The above observations might be the reason why, in this study, Aker induced both angiogenesis and osteogenesis of iPSCs in 3D scaffolds and in vivo.

In bone tissue engineering, angiogenesis is the basic step in the process of bone regeneration, which provides blood supply and guide the subsequent progress of osteogenesis. ${ }^{68}$ Angiogenesis occurs before osteogenesis in the healing of bone defects, and afterwards, both angiogenesis and osteogenesis 


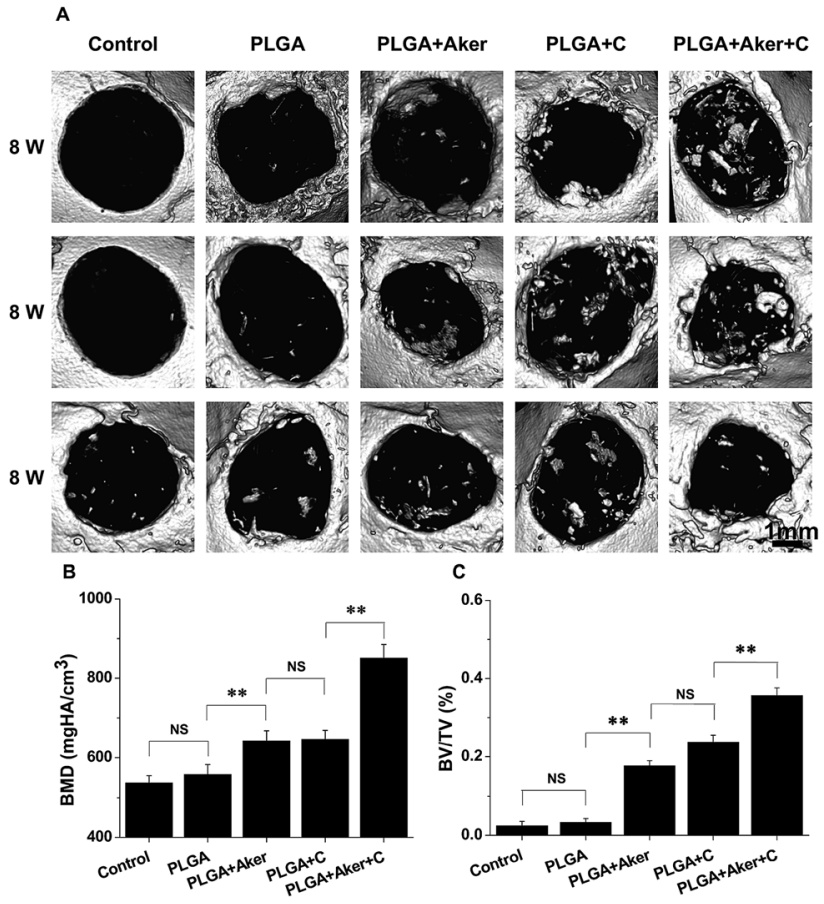

Fig. 6 Analysis of new bone formation in skull defects at 8 weeks post-operation. (A) Microcomputer tomography (micro-CT) images of skull defects were detected after implantation at 8 weeks. Scale bar $=1$ $\mathrm{mm}$. (B) Local bone mineral density (BMD) analysis by micro-CT of five groups at 8 weeks post-operation. (C) Morphometric analysis (BV/TV) of new bone formation. Quantitative micro-CT analysis revealed that implantation of PLGA + Aker + C group achieved the highest local $B M D$ and the highest amount of new bone formation than other four groups. ${ }^{* *} p<0.01$. NS: no significance. Scale bar $=1 \mathrm{~mm}$.

participate in bone regeneration and promote the effect of each other. ${ }^{69}$ Moreover, previous studies reported that the osteogenesis and angiogenesis of BMSCs and HUVECs are inducible by porous $\beta$-CaSiO ${ }_{3} /$ PDLGA composite scaffold via the activation of AMPK/ERK1/2 and PI3K/AKT pathways. ${ }^{16}$ Reports also indicate that the mitogen-activated protein kinases (MAPKs) including the extracellular signal-regulated kinase $(\mathrm{ERK})^{70,71}$ and the AKT signaling pathway play important roles in the osteogenic differentiation of progenitor cells as well as the expression of angiogenic factors. ${ }^{72-74}$ More importantly, a recent study showed that the osteogenesis and angiogenesis effect of Aker bioceramics on OVX-BMSCs might be related to P38, ERK, AKT and STAT3 signaling pathways and must be driven by a crosstalk regulation among these signaling pathways. ${ }^{75}$ Therefore, we speculate that the stimulation angiogenesis and osteogenesis of iPSCs by Aker might also be related to P38, ERK, AKT and STAT3 signaling pathways. Thus, further experiments are required in the future to confirm our speculation and explore the exact mechanism by which the engineered composite drive the repair of bone defects.

\section{Conclusions}

In the present work, we demonstrated that PLGA/Aker 3D composite scaffolds could stimulate the osteogenic and angiogenic differentiation of human iPSCs. In vivo, PLGA/+10\% Aker scaffolds induced the vascularization and osteogenic differentiation of human iPSCs in subcutaneous tissue and effectively promoted bone regeneration in critical-sized calvarial defects. Our work suggests that PLGA/+10\% Aker 3D composite scaffolds are promising candidates for bone tissue engineering.

\section{Conflicts of interest}

There are no conflicts to declare.

\section{Acknowledgements}

This work was supported by the Natural Science Foundation of China (Grant No. 31271054 and Grant No. 31770980).

\section{Notes and references}

1 C. Bock, E. Kiskinis, G. Verstappen, H. Gu, G. Boulting, Z. D. Smith, M. Ziller, G. F. Croft, M. W. Amoroso, D. H. Oakley, A. Gnirke, K. Eggan and A. Meissner, Cell, 2011, 144, 439-452.

2 K. Takahashi, K. Tanabe, M. Ohnuki, M. Narita, T. Ichisaka, K. Tomoda and S. Yamanaka, Cell, 2007, 131, 861-872.

3 L. Warren, P. D. Manos, T. Ahfeldt, Y.-H. Loh, H. Li, F. Lau, W. Ebina, P. K. Mandal, Z. D. Smith, A. Meissner, G. Q. Daley, A. S. Brack, J. J. Collins, C. Cowan, T. M. Schlaeger and D. J. Rossi, Cell Stem Cell, 2010, 7, 618-630.

4 G. Bilousova, D. H. Jun, K. B. King, S. De Langhe, W. S. Chick, E. C. Torchia, K. S. Chow, D. J. Klemm, D. R. Roop and S. M. Majka, Stem Cells, 2011, 29, 206-216.

5 J. H. Ye, Y. J. Xu, J. Gao, S. G. Yan, J. Zhao, Q. Tu, J. Zhang, X. J. Duan, C. A. Sommer, G. Mostoslavsky, D. L. Kaplan, Y. N. Wu, C. P. Zhang, L. Wang and J. Chen, Biomaterials, 2011, 32, 5065-5076.

6 J. Xie, C. Peng, Q. Zhao, X. Wang, H. Yuan, L. Yang, K. Li, X. Lou and Y. Zhang, Acta Biomater., 2016, 29, 365-379.

7 D. Sheyn, S. Ben-David, G. Shapiro, S. De Mel, M. Bez, L. Ornelas, A. Sahabian, D. Sareen, X. Da, G. Pelled, W. Tawackoli, Z. Liu, D. Gazit and Z. Gazit, Stem Cells Transl. Med., 2016, 5, 1447-1460.

8 X. Dong, H. Li, Y. Zhou, L. Ou, J. Cao and J. Chang, J. Mater. Chem. B, 2016, 4, 2369-2376.

9 H. H. Lu, S. F. El-Amin, K. D. Scott and C. T. Laurencin, J. Biomed. Mater. Res., Part A, 2003, 64, 465-474.

$10 \mathrm{H}$. Li and J. Chang, J. Mater. Sci.: Mater. Med., 2004, 15, 10891095.

11 H. Li, W. Zhai and J. Chang, J. Biomater. Appl., 2009, 24, 231246.

12 W. Cheng, H. Li and J. Chang, Mater. Lett., 2005, 59, 22142218.

13 H. Li, R. Du and J. Chang, J. Biomater. Appl., 2005, 20, 137155.

14 H. Li and J. Chang, Compos. Sci. Technol., 2005, 65, 22262232. 
15 H. Li, K. Xue, N. Kong, K. Liu and J. Chang, Biomaterials, 2014, 35, 3803-3818.

16 C. Wang, K. Lin, J. Chang and J. Sun, Biomaterials, 2013, 34, 64-77.

17 C. Wu, D. Zhai, H. Ma, X. Li, Y. Zhang, Y. Zhou, Y. Luo, Y. Wang, Y. Xiao and J. Chang, Acta Biomater., 2014, 10, 3295-3306.

18 S. J. Peter, M. J. Miller, A. W. Yasko, M. J. Yaszemski and A. G. Mikos, J. Biomed. Mater. Res., 1998, 43, 422-427.

19 H. Kim, H. M. Kim, J. E. Jang, C. M. Kim, E. Y. Kim, D. Lee and G. Khang, Int. J. Stem Cells, 2013, 6, 67.

20 J. S. Carson and M. P. Bostrom, Injury, 2007, 38(suppl. 1), S33-S37.

21 S. Xu, K. Lin, Z. Wang, J. Chang, L. Wang, J. Lu and C. Ning, Biomaterials, 2008, 29, 2588-2596.

22 M. Zhang, C. Wu, K. Lin, W. Fan, L. Chen, Y. Xiao and J. Chang, J. Biomed. Mater. Res., Part A, 2012, 100, 2979-2990.

23 A. Hoppe, N. S. Güldal and A. R. Boccaccini, Biomaterials, 2011, 32, 2757-2774.

$24 \mathrm{H}$. Li and J. Chang, Acta Biomater., 2013, 9, 5379-5389.

25 H. Li and J. Chang, Acta Biomater., 2013, 9, 6981-6991.

26 A. A. Gorustovich, J. A. Roether and A. R. Boccaccini, Tissue Eng., Part B, 2010, 16, 199-207.

27 R. M. Day, Tissue Eng., 2005, 11, 768-777.

28 L. C. Gerhardt, K. L. Widdows, M. M. Erol, C. W. Burch, J. A. Sanz-Herrera, I. Ochoa, R. Stampfli, I. S. Roqan, S. Gabe, T. Ansari and A. R. Boccaccini, Biomaterials, 2011, 32, 4096-4108.

29 W. Zhai, H. Lu, C. Wu, L. Chen, X. Lin, K. Naoki, G. Chen and J. Chang, Acta Biomater., 2013, 9, 8004-8014.

30 W. Zhai, H. Lu, L. Chen, X. Lin, Y. Huang, K. Dai, K. Naoki, G. Chen and J. Chang, Acta Biomater., 2012, 8, 341-349.

31 X. Huang, X. Zhang, X. Wang, C. Wang and B. Tang, J. Biosci. Bioeng., 2012, 114, 1-8.

32 C. Wu and J. Chang, J. Biomater. Appl., 2006, 21, 119-129.

33 R. Tokita, K. Nakajima, K. Inoue, A. Al-Wahabi, T. Ser-Od, K. Matsuzaka and T. Inoue, Dent. Mater. J., 2017, 36, 103110.

34 S. H. Oh, I. K. Park, J. M. Kim and J. H. Lee, Biomaterials, 2007, 28, 1664-1671.

35 D. Kaigler, P. H. Krebsbach, E. R. West, K. Horger, Y.-C. Huang and D. J. Mooney, FASEB J., 2005, 19, 665-667.

36 C. M. Stanford, P. A. Jacobson, E. D. Eanes, L. A. Lembke and R. J. Midura, J. Biol. Chem., 1995, 270, 9420-9428.

37 J. S. Son, M. Appleford, J. L. Ong, J. C. Wenke, J. M. Kim, S. H. Choi and D. S. Oh, J. Controlled Release, 2011, 153, 133-140.

38 A. R. Shrivats, M. C. McDermott and J. O. Hollinger, Drug Discovery Today, 2014, 19, 781-786.

39 A. Ardeshirylajimi, J. Cell. Biochem., 2017, 118, 3034-3042.

40 A. R. Amini, C. T. Laurencin and S. P. Nukavarapu, Crit. Rev. Biomed. Eng., 2012, 40, 363-408.

41 D. Marolt, I. M. Campos, S. Bhumiratana, A. Koren, P. Petridis, G. Zhang, P. F. Spitalnik, W. L. Grayson and G. Vunjak-Novakovic, Proc. Natl. Acad. Sci. U. S. A., 2012, 109, 8705-8709.
42 A. Kanani and S. Bahrami, Trends Biomater. Artif. Organs, 2010, 24, 93-115.

43 Y. Han, Q. Zeng, H. Li and J. Chang, Acta Biomater., 2013, 9, 9107-9117.

44 K. Lin, L. Xia, H. Li, X. Jiang, H. Pan, Y. Xu, W. W. Lu, Z. Zhang and J. Chang, Biomaterials, 2013, 34, 10028-10042.

45 J. Guan, J. Zhang, S. Guo, H. Zhu, Z. Zhu, H. Li, Y. Wang, C. Zhang and J. Chang, Biomaterials, 2015, 55, 1-11.

46 Y. Huang, X. Jin, X. Zhang, H. Sun, J. Tu, T. Tang, J. Chang and K. Dai, Biomaterials, 2009, 30, 5041-5048.

47 H. Sun, C. Wu, K. Dai, J. Chang and T. Tang, Biomaterials, 2006, 27, 5651-5657.

48 Q. Liu, L. Cen, S. Yin, L. Chen, G. Liu, J. Chang and L. Cui, Biomaterials, 2008, 29, 4792-4799.

49 H. Gu, F. Guo, X. Zhou, L. Gong, Y. Zhang, W. Zhai, L. Chen, L. Cen, S. Yin, J. Chang and L. Cui, Biomaterials, 2011, 32, 7023-7033.

50 H. Schliephake, F. W. Neukam, D. Hutmacher and J. Becker, J. Oral Maxillofac. Surg., 1994, 52, 57-63.

51 V. Maquet and R. Jerome, Mater. Sci. Forum, 1997, 250, 1542.

52 C. M. Agrawal, K. A. Athanasiou and J. D. Heckman, Mater. Sci. Forum, 1997, 250, 115-128.

53 C. M. Agrawal and R. B. Ray, J. Biomed. Mater. Res., 2001, 55, 141-150.

54 J. M. Diaz-Tocados, C. Herencia, J. M. Martinez-Moreno, A. Montes de Oca, M. E. Rodriguez-Ortiz, N. Vergara, A. Blanco, S. Steppan, Y. Almaden, M. Rodriguez and J. R. Munoz-Castaneda, Sci. Rep., 2017, 7, 7839.

55 D. Bernardini, Front. Biosci., 2005, 10, 1177-1182.

56 S. Yoshizawa, A. Brown, A. Barchowsky and C. Sfeir, Acta Biomater., 2014, 10, 2834-2842.

57 J. A. Maier, D. Bernardini, Y. Rayssiguier and A. Mazur, Biochim. Biophys. Acta, 2004, 1689, 6-12.

58 S. L. Natividad-Diaz, S. Browne, A. K. Jha, Z. Ma, S. Hossainy, Y. K. Kurokawa, S. C. George and K. E. Healy, Biomaterials, 2019, 194, 73-83.

59 J. R. Bezenah, A. Y. Rioja, B. Juliar, N. Friend and A. J. Putnam, Biotechnol. Bioeng., 2019, 116, 415-426.

60 W. Lin, M. Chen, C. Hu, S. Qin, C. Chu, L. Xiang, Y. Man and Y. Qu, BioMed Res. Int., 2018, 2018, 8459503.

61 Z. E. Clayton, G. S. Yuen, S. Sadeghipour, J. D. Hywood, J. W. Wong, N. F. Huang, M. K. Ng, J. P. Cooke and S. Patel, Int. J. Cardiol., 2017, 234, 81-89.

62 A. J. Rufaihah, N. F. Huang, S. Jame, J. C. Lee, H. N. Nguyen, B. Byers, A. De, J. Okogbaa, M. Rollins, R. Reijo-Pera, S. S. Gambhir and J. P. Cooke, Arterioscler. Thromb. Vasc. Biol., 2011, 31, e72-79.

63 C. H. Yoo, H. J. Na, D. S. Lee, S. C. Heo, Y. An, J. Cha, C. Choi, J. H. Kim, J. C. Park and Y. S. Cho, Biomaterials, 2013, 34, 8149-8160.

64 J. Liang, W. Huang, W. Cai, L. Wang, L. Guo, C. Paul, X. Y. Yu and Y. Wang, Stem Cells, 2017, 35, 337-350.

65 R. P. Tan, A. H. P. Chan, K. Lennartsson, M. M. Miravet, B. S. L. Lee, J. Rnjak-Kovacina, Z. E. Clayton, J. P. Cooke, M. K. C. Ng, S. Patel and S. G. Wise, Stem Cell Res. Ther., 2018, 9, 70 . 
66 J. J. Kim, L. Hou, G. Yang, N. P. Mezak, M. Wanjare, L. M. Joubert and N. F. Huang, Cell. Mol. Bioeng., 2017, 10, 417-432.

67 T. Chen, A. Margariti, S. Kelaini, A. Cochrane, S. T. Guha, Y. Hu, A. W. Stitt, L. Zhang and Q. Xu, Stem Cells, 2015, 33, 1405-1418.

68 H. Winet, Bone, 1996, 19, S39-S57.

69 J. Glowacki, Clin. Orthop. Relat. Res., 1998, S82-S89.

70 Y. Wu, L. Xia, Y. Zhou, Y. Xu and X. Jiang, Cell Proliferation, 2015, 48, 375-384.
71 Y. Zhou, Y. Wu, X. Jiang, X. Zhang, L. Xia, K. Lin and Y. Xu, PLoS One, 2015, 10, e0129605.

72 S. P. Ayalasomayajula and U. B. Kompella, Eur. J. Pharmacol., 2002, 449, 213-220.

73 J. Chen, R. Crawford, C. Chen and Y. Xiao, Tissue Eng., Part B, 2013, 19, 516-528.

74 L. L. Chen, M. Huang, J. Y. Tan, X. T. Chen, L. H. Lei, Y. M. Wu and D. Y. Zhang, Tissue Eng., Part A, 2013, 19, 2226-2232.

75 L. Xia, Z. Yin, L. Mao, X. Wang, J. Liu, X. Jiang, Z. Zhang, K. Lin, J. Chang and B. Fang, Sci. Rep., 2016, 6, 22005. 